\title{
Mechanism of a Disassembly Driven Sensing System Studied by Stopped-Flow Kinetics.
}

\author{
Cara Gallo, Suma S. Thomas, Allison J. Selinger, Fraser Hof* and Cornelia Bohne* \\ Department of Chemistry and Centre for Advanced Materials and Related Technologies (CAMTEC), University of Victoria, \\ PO Box 1700 STN CSC, Victoria, BC V8W 2Y2, Canada
}

\begin{abstract}
We carried out steady-state and stopped-flow photophysical measurements to determine the kinetics of a discrete disassembly driven turn-on fluorescent system. On and off rates for both DimerDye1 (DD1) assembly, and nicotine binding were determined. Relative rates for these competing processes provide insight on how this system can be optimized for sensing applications. Kinetics studies in artificial saliva showed that moving to more complex media has minimal effects on the sensing ability of the system.
\end{abstract}

In supramolecular sensing, the coupling of a molecular recognition event to a fluorescent output can happen in many ways. ${ }^{1-5}$ Analyte-triggered disaggregation is a broadly successful approach for turn-on fluorescence sensing. ${ }^{6-8}$ Amphiphilic dendrimers and polymers - easily functionalized and intrinsically suited to forming large aggregates — are often used for disassembly-driven sensing. ${ }^{9-13}$ Host-guest binding can also be used to trigger the disassembly of molecular micelles, in order to provide visible responses. Where a host-indicator complex forms large, quenched micellar aggregates, the presence of a guest-type analyte can cause disassembly and render the indicator emissive. ${ }^{14}$ This approach is operational in complex biological and aqueous media, and has been used as a general approach for selectively sensing drugs, ${ }^{15-16}$ and biologically relevant analytes. ${ }^{17-19}$

Mechanistic details about dynamic phenomena are germane for the design of complex systems at equilibrium or out-ofequilbrium. ${ }^{5,20-22}$ Studies for tandem assays based on host-guest disassembly showed that disassembly must be faster than the assayed reaction. ${ }^{23}$ The micellar or polymeric nature of many disassembly-driven sensors complicates their mechanistic characterization. ${ }^{24}$ In contrast, knowledge on how to control sequential and competitive pathways for stoichiometric disassembly systems will provide the information required for the optimization of such systems.

Scheme 1. Fluorescence turn-on of the HSP moiety of DD1 with the binding of nicotine guest.
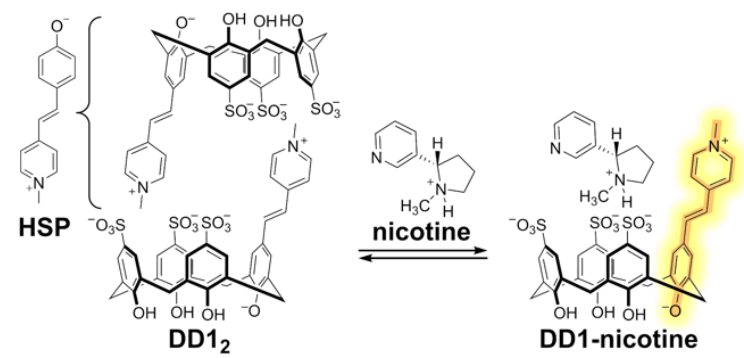

We have previously reported a series of calixarenes that homodimerize in water. ${ }^{25}$ Analogs that include an integrated fluorescent component are called DimerDyes. ${ }^{26-27}$ The self-assembly of DimerDyes stacks two dye copies in an antiparallel, quenching arrangement. The addition of a good guest out-competes homodimerization to form a host-guest complex, resulting in a turn-on fluorescence response (Scheme 1). These disassembly-driven systems are inherently resistant to interference from competing co-solutes, ${ }^{28}$ making them well suited to many biosensing tasks. As discrete assemblies, DimerDyes are uniquely well suited to detailed mechanistic characterization.

The current study focuses on the competing binding equilibria for the complexes of DimerDye1 (DD1) with the guest nicotine. Nicotine is a biologically relevant model analyte ${ }^{27,29}$ that has multiple ionizable groups $\left(\mathrm{p} K_{\mathrm{a}}=3.1\right.$ (pyridine) and 8.0 (pyrrolidine) $),{ }^{30}$ allowing us to probe the effect of charge on multiple binding equilibria. We first characterize the general assembly and photophysical properties of the system using absorbance and steady-state fluorescence. We then used stopped-flow experiments in buffer and artificial saliva to establish the kinetics of individual steps, and to reveal how complex media affect the kinetics at the heart of this disassembly-driven sensor.

Dimerization of DD1 is affected by the protonation states of the host. Sulfonatocalix[4]arenes have a single, unusually acidic phenol with a $\mathrm{p} K_{\mathrm{a}}$ of 3.2 , while the remaining three phenols have $\mathrm{p} K_{\mathrm{a}}$ values $\geq 11.3$. $^{31-32}$ At $\mathrm{pH} 6.3$ (one anionic phenol, Scheme 1) and at $\mathrm{pH} 12.1$ ( $>1$ anionic phenol) DD1 exists as the non-fluorescent dimeric $\mathrm{DD}_{2}$ (inset Figure $1 \mathrm{a}, \mathrm{b}$ ). ${ }^{26}$ At $\mathrm{pH}$ 2.2, some fluorescence is observed (Figure 1b), indicating the presence of the fluorescent monomer DD1. This weakening of the dimer is likely due to the $\mathrm{pH}$ being lower than the $\mathrm{p} K_{\mathrm{a}}$ of the hydroxyl group on the lower rim of DD1. 


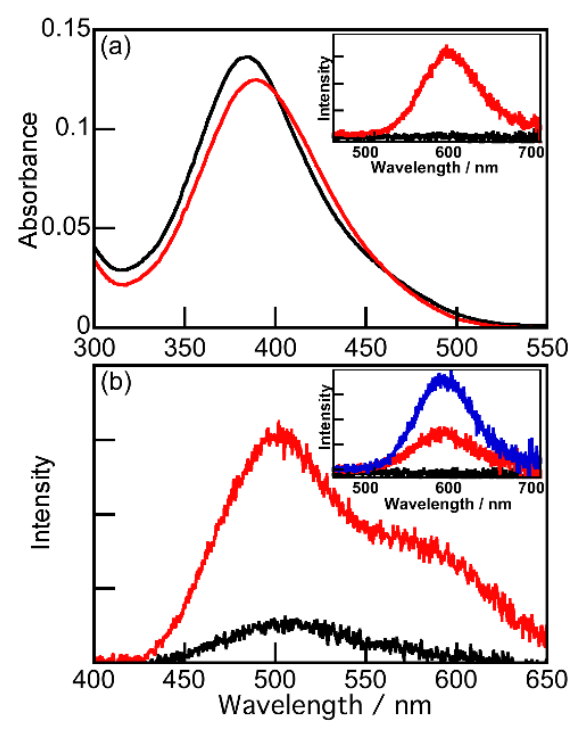

Figure 1. (a) Absorption and fluorescence (inset) spectra for $8.3 \mu \mathrm{M}$ $\mathrm{DD} 1$ in the absence (black) and presence (red) of $167 \mu \mathrm{M}$ nicotine at $\mathrm{pH}$ 6.3. (b) Fluorescence spectra for $8.3 \mu \mathrm{M}$ DD1 in the absence (black) and presence of $167 \mu \mathrm{M}$ nicotine (red) at $\mathrm{pH}$ 2.2. Inset: Fluorescence spectra for $10 \mu \mathrm{M}$ DD1 in the absence (black) and presence of 500 (red) and $2000 \mu \mathrm{M}$ (blue) choline at $\mathrm{pH} 12.1$.

Optimal DD1-guest binding occurs with positively charged guests. Upon treatment with nicotine at $\mathrm{pH} 6.3$ and 2.2, a red shift in the absorption spectra and increase in emission intensities are observed (Figure 1, S2). This is expected to arise from the disassembly of $\mathrm{DD}_{2}$ and formation of the DD1-nicotine complex. The existence of some disassembled DD1 at $\mathrm{pH} 2.2$ leads to detectable fluorescence in the absence of nicotine, which signifies the presence of a mixture of species. At $\mathrm{pH} 12.1$, nicotine is uncharged; the minimal response of DD1 absorbance and emission upon addition of nicotine (Figure S3) confirms that a cationic guest is required for significant binding to occur. ${ }^{29}$ This is verified by treatment of DD1 with the quaternary ammonium ion choline at $\mathrm{pH}$ 12.1, for which a fluorescence response occurs (inset Fig, 1b). Sensing at various $\mathrm{pH}$ values will be instrumental for the simultaneous sensing of multiple analytes that have differentially charged states at the different pHs. ${ }^{33-34}$ However, for our kinetic studies we chose the $\mathrm{pH}$ of 6.3 where we have the presence of only one well behaved charge state of host and guest.

The DD1-nicotine system corresponds to a system of coupled reactions requiring real-time measurement of its kinetics. The association and dissociation processes cannot be determined separately and the fastest unimolecular reaction determines the time scale for the kinetics measurements. ${ }^{35}$ The broadening observed in the ${ }^{1} \mathrm{H}$ NMR spectra for DimerDye-guest systems, ${ }^{26-}$ ${ }^{27}$ suggests that the dynamics for these systems occur on the millisecond time-scale, making stopped-flow the suitable technique for real-time kinetic measurements. ${ }^{35}$

The kinetics for $\mathrm{DD}_{2}$ disassembly upon dilution was measured by the appearance of fluorescence from monomeric DD1 (Figure 2b). No photoisomerization of the DD1 chromophore (HSP) occurred (Figure S4), consistent with the much slower photoisomerization for HSP observed previously. ${ }^{36}$ At higher DD1 concentrations, the reaction is faster because of a faster rate for DD1 association to form $\mathrm{DD}_{2}$. The kinetics follow a monoexponential function from which an observed rate constant $\left(k_{\mathrm{obs}}\right)$ is determined. The linear dependence (eq 1$)^{37}$ between $k_{\mathrm{obs}}^{2}$ and the total DD1 concentration (Figure 2c) led to the determination of the association $\left(k_{\mathrm{as}}\right)$ and the dissociation $\left(k_{\mathrm{dis}}\right)$ rate constants (Table 1$)$. The ratio of $k_{\mathrm{as}}$ and $k_{\mathrm{dis}}$ corresponds to the equilibrium constant for dimerization $\left(K_{\mathrm{DD} 1}\right)$ of $(1.6 \pm 0.9) \times 10^{5} \mathrm{M}^{-1}$, a value that is similar to $K_{\mathrm{DD} 1}$ determined from ${ }^{1} \mathrm{H}$ NMR experiments in $\mathrm{D}_{2} \mathrm{O}(3.6 \pm 0.8) \times 10^{5} \mathrm{M}^{-1} .^{26}$
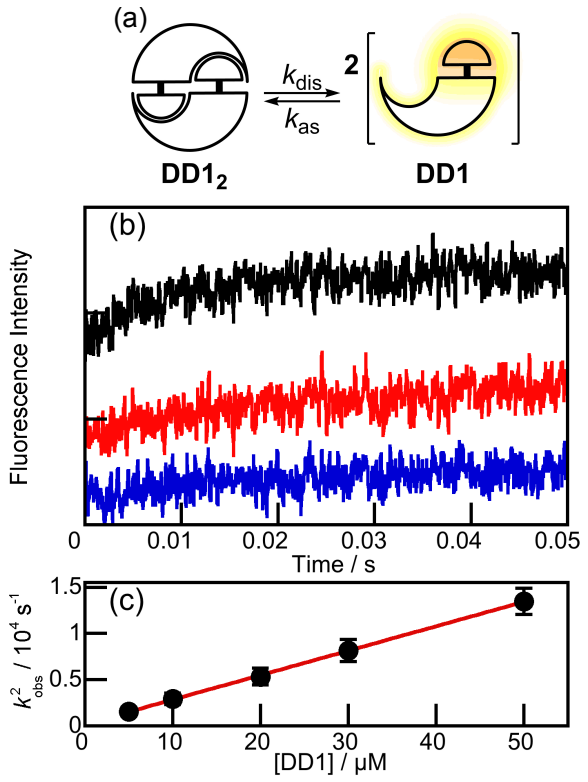

Figure 2. (a) Schematics for the dimerization of DD1. (b) Kinetics for the dilution of DD1 ([DD1 $]_{\text {final }}$ : blue $-5 \mu \mathrm{M}$, red $-10 \mu \mathrm{M}$, black $-50 \mu \mathrm{M}$ ) with phosphate buffer at $\mathrm{pH}$ 6.3. The traces are offset on the y-scale for clarity. (c) Fit to equation 1 for the dependence of $k_{o b s}^{2}$ ( 7 independent experiments; errors correspond to standard deviations) on the DD1 concentration.

$$
k_{\mathrm{obs}}^{2}=k_{\mathrm{dis}}^{2}+8 k_{\mathrm{as}}[\mathrm{DD} 1]_{\mathrm{tot}}
$$

Table 1. Association and dissociation rate constants for the dimerization of DD1 and the DD1-nicotine complex, and equilibrium constants for $\mathrm{DD1}_{2}\left(K_{\mathrm{DD} 1}\right)$ and DDI-Nic $\left(K_{11}\right)$ in buffer at pH 6.3 or in artificial saliva. ${ }^{a}$

\begin{tabular}{ccccc}
\hline & \multicolumn{2}{c}{ DD1 } & \multicolumn{2}{c}{ DD1-nicotine } \\
\cline { 2 - 5 } & Buffer & Saliva & Buffer & Saliva \\
\hline$k_{a s} / 10^{6} \mathrm{M}^{-1} \mathrm{~s}^{-1}$ & $2.3 \pm 0.9$ & $2 \pm 1$ & & \\
$k_{d i s} / \mathrm{s}^{-1}$ & $14 \pm 6$ & $80 \pm 40$ & & \\
$K_{\mathrm{DD} 1} / 10^{4} \mathrm{M}^{-1} \mathrm{~b}$ & $16 \pm 9$ & $3 \pm 2$ & & \\
$k_{11}^{+} / 10^{6} \mathrm{M}^{-1} \mathrm{~s}^{-1}$ & & & $0.23 \pm 0.01$ & $0.10 \pm 0.02$ \\
$k_{11}^{-} / \mathrm{s}^{-1}$ & & & $40 \pm 10$ & $150 \pm 30$ \\
$K_{11} / 10^{4} \mathrm{M}^{-1 \mathrm{~b}}$ & & & $0.5 \pm 0.1$ & $0.07 \pm 0.02$ \\
$K_{11} / 10^{4} \mathrm{M}^{-1 \mathrm{c}}$ & & & $0.6 \pm 0.1$ & $0.13 \pm 0.02$ \\
\hline
\end{tabular}

${ }^{\mathrm{a}}$ For error determinations see the Supporting Information. ${ }^{\mathrm{b}} \mathrm{Cal}-$ culated from kinetic data, where $K_{11}=k_{11}^{+} / k_{11}^{-}$. ${ }^{\mathrm{c}}$ Obtained from the binding isotherm.

The association rate constant for $\mathrm{DD}_{2}$ formation suggests that dimerization requires some rearrangement of the macrocycle to accommodate the HSP moiety, since $k_{\text {as }}$ is smaller than the diffusion-controlled limit $\left(6 \times 10^{9} \mathrm{M}^{-1} \mathrm{~s}^{-1}\right)^{38}$ and smaller 
than the association rate constant of a neutral radical with $p$ sulfonatocalix[4]arene. ${ }^{39}$ This moderate decrease of $k_{\text {as }}$ for $\mathrm{DD}_{2}$ suggests that rearrangement and loss of entropy are required for dimerization to occur. However, no significant restrictions exist. Such restrictions, when observed for other macrocycles, like cyclodextrins and cucurbit $[n]$ urils, lead to slow complexation that can take hours. ${ }^{40-44}$ The dissociation rate constant for $\mathrm{DD}_{2}$ disassembly is similar to that for unimpeded guest dissociation from cucurbit $[n]$ urils, ${ }^{45-46}$ but much slower than the dissociation of guests from cyclodextrins. ${ }^{43}$ This slow disassembly off-sets the low association rate constant leading to an equilibrium constant that ensures that micromolar concentrations of DD1 can be used for sensing applications.

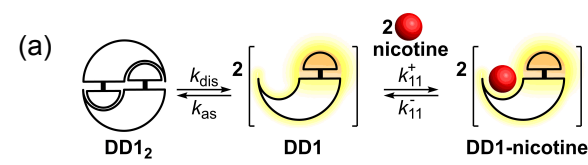

(b)

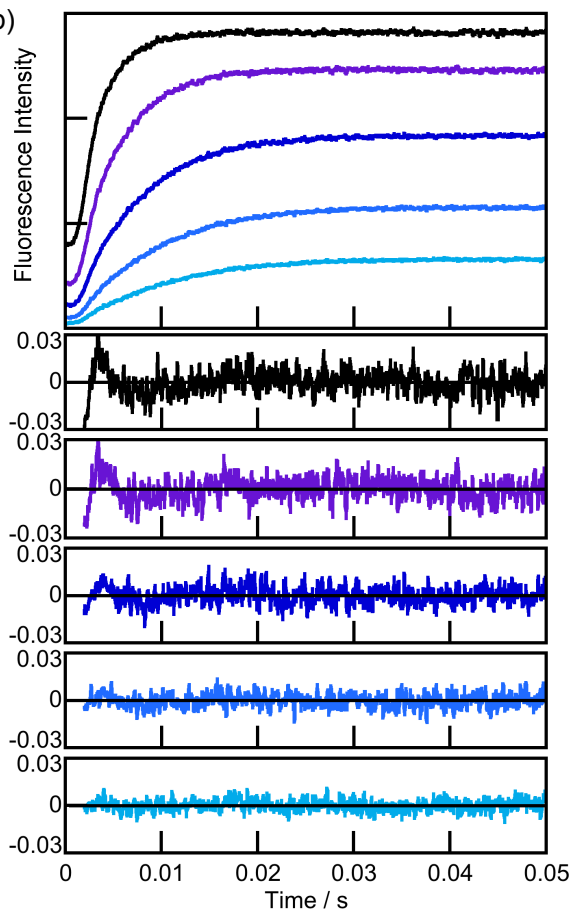

Figure 3. (a) Schematics for DD1 dimerization and nicotine binding to DD1. (b) Kinetics for the mixing of DD1 $(10 \mu \mathrm{M}, \mathrm{pH} 6.3)$ with different concentrations of nicotine for one independent experiment. Residuals obtained for the data from the global analysis of 21 kinetic traces from four independent experiments. From the bottom to top: $[\text { nicotine }]_{\text {final }}=94,188,375,750$ and $1500 \mu \mathrm{M}$.

Differences in the association and dissociation rate constants for the complexation dynamics of DD1-nicotine and DD1 2 provide a guide for future optimization of nicotine sensing. Sensing is not optimal because with the low $K_{11}$ for DD1-nicotine a high nicotine concentration is required to achieve detectable response. The low $K_{11}$ compared to $K_{\mathrm{DD} 1}$ is a consequence of the lower value of $k_{11}^{+}$compared to $k_{\text {as }}$ and the higher value of $k_{11}^{-}$compared to $k_{\text {dis, }}$, where the larger change for $k_{11}^{+}$has a larger effect on $K_{11}$. The best target for host modification will be to increase the association rate constant $k_{11}^{+}$in order for nicotine association to outcompete DD1 dimerization. Decreasing the nicotine dissociation rate constant would also achieve a higher
$K_{11}$ value, but too slow a nicotine dissociation rate might interfere when sensing for multiple targets is desirable.

The kinetics were also studied in artificial saliva ${ }^{47}$ at $\mathrm{pH} 6.8$ to understand how a complex environment mimicking a biological one affects the mechanism of the DD1 system. The kinetics for both equilibria were qualitatively the same as in buffer (Figures S6-7). The $k_{\text {as }}$ for $\mathrm{DD}_{2}$ dimerization remained the same, while the rate constant for disassembly increased (Table 1). Cations are known to bind to $p$-sulfonatocalix[4]arene ${ }^{48-49}$ and have been shown to modulate the kinetics for guest binding to a calix[6]arene in organic solvents. ${ }^{50}$ The higher $k_{\text {dis }}$ for $\mathrm{DD} 1_{2}$ disassembly suggests that cations stabilize the monomeric DD1, while the unchanged $k_{\text {as }}$ value indicates that dimerization is not inhibited by cation binding to DD1, ensuring that the decrease of $K_{\mathrm{DD} 1}$ is only modest. The trends for the dynamics of DD1nicotine were the same as in buffer with a further decrease observed for $k_{11}^{+}$and a more significant increase observed for $k_{11}^{-}$. From the mechanistic point of view the relative rate constant for $\mathrm{DD}_{2}{ }_{2}$ dimerization and DD1-nicotine complexation did not change significantly, showing that mechanistic kinetic studies in buffer provide relevant information for the design of the next generation of disassembly driven sensors.

\section{ASSOCIATED CONTENT}

\section{Supporting Information}

The Supporting Information is available.

Experimental section, absorption and fluorescence spectra at different $\mathrm{pHs}$, DD1 photostability studies, global analysis on the DD1nicotine kinetics, binding isotherm for DD1-nicotine and kinetics for DD1-nicotine in artificial saliva.

\section{AUTHOR INFORMATION}

\section{Corresponding Author}

Fraser Hof - Department of Chemistry and Centre for Advanced Materials and Related Technologies (CAMTEC), University of Victoria, Victoria, BC V8W 2Y2, Canada; Email: fhof@uvic.ca. Cornelia Bohne - Department of Chemistry and Centre for Advanced Materials and Related Technologies (CAMTEC), University of Victoria, Victoria, BC V8W 2Y2, Canada; Email: cornelia.bohne@gmail.com

\section{Author Contributions}

Cara Gallo: Investigation, formal data analysis. Suma S. Thomas: Formal analysis, supervision, writing, visualization. Allison J.

Selinger: Supervision, writing, visualization. Fraser Hof: Conceptualization, writing, review and editing. Cornelia Bohne: Conceptualization, writing, review and editing

Notes

The authors declare no competing financial interest.

\section{ACKNOWLEDGMENT}

The authors thank the Natural Sciences and Engineering Research Council of Canada (NSERC) for financial support (CB-RGPIN2017-04458; FH-RGPIN-2019-04806) and CAMTEC for the use of shared facilities.

\section{REFERENCES}

1. Sedwick, A. C.; Brewster II, J. T.; Wu, T.; Feng, X.; Bull, S. D.; Qian, X.; Sessler, J. L.; T.D., J.; Anslyn, E. V.; Sun, X., Indicator 
displacement assays (IDAs): the past, present and future. Chem. Soc. Rev. 2021, 50, 9-38.

2. Guo, C.; Sedwick, A. C.; T., H.; Sessler, J. L., Supramolecular fluorescent sensors: An historical overview and update. Coord. Chem. Rev. 2021, 427, 213560.

3. Pinalli, R.; Pedrini, A.; Dalcanale, R., Biochemical sensing with macrocyclic receptors. Chem. Soc. Rev. 2018, 47, 7006-7026.

4. $\quad$ Kumar, R.; Sharma, A.; Singh, H.; Suating, P.; Kim, H. S.; Sumwoo, K.; Shim, I.; Gibb, B. C.; Kim, J. S., Revisiting Fluorescent Calixarenes: From Molecular Sensors to Smart Materials. Chem. Rev. 2019, 119, 9657-9721.

5. Ghale, G.; Nau, W. M., Dynamically Analyte-Responsive Macrocyclic Host-Fluorophore Systems. Acc. Chem. Res. 2014, 47, 2150-2159.

6. Mizusawa, K.; Ishida, Y.; Takaoka, Y.; Miyagawa, M.; Tsukiji, S.; Hamachi, I., Disassembly-Driven Turn-On Fluorescent Nanoprobes for Selective Protein Detection. J. Am. Chem Soc. 2010, 132, 7291-7293.

7. Bo, F.; Gao, B.; Duan, W.; Li, H.; Liu, H.; Bai, Q., Assembly-disassembly driven "off-on" fluorescent perylene bisimide probes for detecting and tracking of proteins in living cells. RSC Adv. 2013, 3, 17007-17010.

8. Tian, D.; Li, F.; Zhu, Z.; Zhang, L.; Zhu, J., An AIE-based metallo-supramolecular assembly enabling an indicator displacement assay inside living cells. Chem. Commun. 2018, 54, 8921-8924.

9. $\quad$ Sakai, R.; Nagai, A.; Tago, Y.; Sato, S.; Arai, T.; Satoh, T.; Kakuchi, T., Fluorescence Turn-On Sensing of Anions Based on Disassembly Process of Urea-Functionalized Poly(phenylenebutadiynylene) Aggregates. Macromolecules 2012, 45, 4123-4127.

10. Savariar, E. N.; Ghosh, S.; González, D. C.; Thayumanavan, S., isassembly of Noncovalent Amphiphilic Polymers with Proteins and Utility in Pattern Sensing. J. Am. Chem Soc. 2008, 130, 5416-5417.

11. González, D. C.; Savariar, E. N.; Thayumanavan, S., Fluorescence Patterns from Supramolecular Polymer Assembly and Disassembly for Sensing Metallo- and Nonmetalloproteins. J. Am. Chem Soc. 2009, 131, 7708-7716.

12. Azagarsamy, M. A.; Yesilyurt, V.; Thayumanavan, S., Disassembly of Dendritic Micellar Containers Due to Protein Binding. J. Am. Chem Soc. 2010, 132, 4550-4551.

13. Tanwar, A. S.; Yver, P. K., Fluorescence "Turn-On" Indicator Displacement Assay-Based Sensing of Nitroexplosive 2,4,6Trinitrophenol in Aqueous Media via a Polyelectrolyte and Dye Complex. ACS Omega 2017, 2, 4424-4430.

14. Liu, Y.; Perez, L.; Mettry, M.; Easley, C. J.; Hooley, R. J.; Zhong, W., Self-Aggregating Deep Cavitand Acts as a Fluorescence Displacement Sensor for Lysine Methylation. J. Am. Chem Soc. 2016, 138, 10746-10749.

15. Gill, A. D.; Hickey, B. L.; Zhong, W.; Hooley, R. J., Selective sensing of THC and related metabolites in biofluids by host:guest arrays. Chem. Commun. 2020, 56, 4352-4355.

16. Gill, A. D.; Perez, L.; Salinas, I. N. Q.; Byers, S. R.; Liu, Y.; Hickey, B. L.; Zhong, W.; Hooley, R. J., Selective Array-Based Sensing of Anabolic Steroids in Aqueous Solution by Host-Guest Reporter Complexes. Chem. Eur. J. 2019, 25, 1740-1745.

17. Liu, Y.; Perez, L.; Gill, A. D.; Hooley, R. J.; Zhong, W., Selective Sensing of Phosphorylated Peptides and Monitoring Kinase and Phosphatase Activity with a Supramolecular Tandem Assay. J. Am. Chem Soc. 2018, 140, 13869-13877.

18. Liu, Y.; Perez, L.; Mettry, M.; Gill, A. D.; Byers, S. R.; Easley, C. J.; Bardeen, C. J.; Zhong, W.; Hooley, R. J., Site selective reading of epigenetic markers by a dual-mode synthetic receptor array. Chem. Sci. 2017, 8, 3960-3970.

19. Gill, A. D.; Hickey, B. L.; Wang, S.; Xue, M.; Zhong, W.; Hooley, R. J., Sensing of citrulline modifications in histone peptides by deep cavitand hosts. Chem. Commun. 2019, 55, 13259-13262.

20. Ashkenasy, G.; Hermans, T. M.; Otto, S.; Taylor, A. F., Systems Chemistry. Chem. Soc. Rev. 2017, 46, 2543-2554.

21. Mattia, E.; Otto, S., Supramolecular systems chemistry. Nat. Nanotechnol. 2015, 10, 111-119.
22. Thomas, S. S.; Tang, H.; Bohne, C., Noninnocent Role of $\mathrm{Na}^{+}$Ions in the Binding of the N-Phenyl-2-naphthylammonium Cation as a Ditopic Guest with Cucurbit[7]uril. J. Am. Chem Soc. 2019, 141, 9645-9654.

23. Florea, M.; Kudithipudi, S.; Rei, A.; González-Álvarez, M.J.; Jeltsch, A.; Nau, W. M., A Fluorescence-Based Supramolecular Tandem Assay for Monitoring Lysine Methyltransferase Activity in Homogeneous Solution. Chem. Eur. J. 2012, 18, 3521-3528.

24. Yesilyurt, V.; Ramireddy, R.; Azargarsamy, M. A.; Thayumanavan, S., Accessing Lipophilic Ligands in Dendrimer-Based Amphiphilic Supramolecular Assemblies for Protein-Induced Disassembly. Chem. Eur. J. 2012, 18, 223-229.

25. Beatty, M. A.; Busmann, J. A.; Fagen, N. G.; Garnett, G. A. E.; Hof, F., A clip-like host that undergoes self-assembly and competitive guest-induced disassembly in water. Supramol. Chem. 2019, 31, 101-107.

26. Beatty, M. A.; Borges-González, J.; Sinclair, N. J.; Pye, A. T.; Hof, F., Analyte-Driven Disassembly and Turn-On Fluorescent Sensing in Competitive Biological Media. J. Am. Chem. Soc. 2018, $140,3500-3504$

27. Beatty, M. A.; Selinger, A. J.; Hof, F., Parallel Synthesis and Screening of Supramolecular Chemosensors That Achieve Fluorescent Turn-on Detection of Drugs in Saliva. J. Am. Chem Soc. 2019, 141 , 16763-16771.

28. Beatty, M. A.; Pye, A. T.; Kim, B.; Selinger, A. J.; Hof, F., Using reversible non-covalent and covalent bonds to create assemblies and equilibrating molecular networks that survive 5 molar urea. $\mathrm{Org}$, Biomol. Chem. 2019, 17, 2081-2086.

29. Warmerdam, Z.; Kamba, B. E.; Saurya, A.; Sun, X.; Maguire, M. K.; Hof, F., Calix[4] arene sulfonate hosts selectively modified on the upper rim: a study of nicotine binding strength and geometry. in press https://doi.org/10.1080/10610278.2021.1873991.

30. El-Hellani, A.; El-Hage, R.; Baalbaki, R.; Salman, R.; Talih, S.; Shihadeh, A.; Saliba, N. A., Free-Base and Protonated Nicotine in Electronic Cigarette Liquids and Aerosols. Chem. Res. Toxicol. 2015, 28, 1532-1537.

31. Yoshida, I.; Yamamotu, N.; Fumio, S.; Ishii, D.; Ueno, K.; Shinkai, S., Re-evaluation of the Acid Dissociation Constants of the Hydroxyl Groups in Tetrasodium 25,26,27,28Tetrahydroxycalix[4]arene-5,11,17,23-tetrasulfonate Bull. Chem. Soc. Jpn. 1992, 65, 1012-1015.

32. Hajmalek, M.; Kalili, M. S.; Zare, K.; Zabihi, O., Determination of the acid dissociation constants of the p-sulphonatocalix[4]arene. J. Nanoanalysis 2014, 1, 47-51.

33. Liu, Y.; Perez, L.; Gill, A. D.; Mettry, M.; L., L.; Wang, Y.; Hooley, R. J.; Zhong, W., Site-Selective Sensing of Histone Methylation Enzyme Activity via an Arrayed Supramolecular Tandem Assay. J. Am. Chem Soc. 2017, 139, 10964-10967.

34. Minaker, S. A.; Daze, K. D.; Ma, M. C. F.; Hof, F., Antibody-Free Reading of the Histone Code Using a Simple Chemical Sensor Array. J. Am. Chem Soc. 2012, 134, 11674-11680.

35. Bohne, C., Supramolecular Dynamics. Chem. Soc. Rev 2014, 43, 4037-4050.

36. Tang, H.; Thomas, S. S.; Wolf, L.; Natarajan, P.; Ko, Y. H.; Wilson, J.; Kim, K.; Bohne, C., Nonlinear Dependence on $\mathrm{Na}^{+}$Ions for the Binding Dynamics of Cucurbit[6]uril with the trans-1-Methyl-4-(4hydroxystyryl)pyridinium Cation. J. Phys. Chem. B 2020, 124 (45), 10219-10225.

37. Bernasconi, C. F., Relaxation Kinetics. Academic Press, Inc.: New York, 1976; p 288.

38. Montalti, M.; Credi, A.; Prodi, L.; Gandolfi, M. T., Handbook of Photochemistry. 3rd ed.; CRC Press: Boca Raton, 2006.

39. Franchi; Lucarini, M.; Pedulli, G. F.; Sciotto, D., An EPR Investigation of the Kinetics of Inclusion of a Persistent Radical in Water-Soluble Calix[4]arenes. Angew. Chem. Int. Ed. 2000, 39, 263266.

40. Cramer, F.; Saenger, W.; Spatz, H.-C., Inclusion Compounds. XIX. The Formation of Inclusion Compounds of aCyclodextrin in Aqueous Solutions. Thermodynamics and Kinetics. $J$. Am. Chem. Soc. 1967, 89, 14-20. 
41. Mock, W. L.; Shih, N. Y., Structure and selectivity in hostguest complexes of cucurbituril. J. Org. Chem. 1986, 51 (23), 44404446.

42. Marquez, C.; Nau, W. M., Two Mechanisms of Slow HostGuest Complexation Between Cucurbit[6]uril and Cyclohexylmethylamine: pH-Responsive Supramolecular Kinetics. Angew. Chem. Int. Ed. 2001, 40 (17), 3155-3160.

43. Bohne, C., Supramolecular Dynamics Studied Using Photophysics. Langmuir 2006, 22, 9100-9111.

44. Masson, E.; Raeisi, M.; Kotturi, K., Kinetics Inside, Outside and Through Cucurbiturils. Isr. J. Chem. 2018, 58 (3-4), 413-434.

45. Tang, H.; Fuentealba, D.; Ko, Y. H.; Selvapalam, N.; Kim, K.; Bohne, C., Guest Binding Dynamics with Cucurbit[7]uril in the Presence of Cations. J. Am. Chem. Soc. 2011, 133, 20623-20633.

46. Thomas, S. S.; Bohne, C., Determination of the kinetics underlying the $\mathrm{p} K \mathrm{a}$ shift for the 2 -aminoanthracenium cation binding with cucurbit[7]uril. Faraday Discuss. 2015, 185, 381-398.
47. Alshali, R. Z.; Salim, N. A.; Satterthwaite, J. D.; Silikas, N., Long-term Sorption and Solubility of Bulk-fill and Conventional Resin-composites in Water and Arificial Saliva. J. Dent. 2015, 43, 1511-1518.

48. Basilio, N.; García-Río, L.; Martín-Pastor, M., Counterion Binding in Solutions of p-Sulfonatocalix[4]arene. J. Phys. Chem. B 2010, 114, 7201-7206.

49. Francisco, V.; Piñero, A.; Nau, W. M.; García-Río, L., The "True" Affinities of Metal Cations to p-Sulfonatocalix[4]arene: A Thermodynamic Study at Neutral pH Reveals a Pitfall Due to Salt Effects in Microcalorimetry. Chem. Eur. J. 2013, 19, 17809-12820.

50. Credi, A.; Dumas, S.; Silvi, S.; Venturi, M.; Arduini, A.; Pochini, A.; Secchi, A., Viologen-Calix[6]arene Pseudorotaxanes. IonPair Recognition and Threading/Dethreading Molecular Motions. $J$. Org. Chem. 2004, 69, 5881-5887. 


\title{
Supporting Information
}

\section{Mechanism of a Disassembly Driven Sensing System Studied by Stopped-Flow Kinetics}

\author{
Cara Gallo, Suma S. Thomas, Allison J. Selinger, Fraser Hof* and Cornelia Bohne* \\ Department of Chemistry and Centre for Advanced Materials and Related Technologies \\ (CAMTEC), University of Victoria, PO Box 1700 STN CSC, Victoria, BC V8W 2Y2, Canada
}

\section{INDEX}

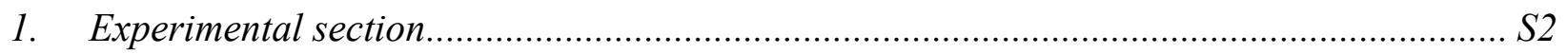

2. Absorption and fluorescence measurements of DD1-nicotine at $p H 2.2$ and $12.1 \ldots \ldots \ldots \ldots . . .54$

3. Control experiment for DD1 photostability during stopped-flow experiments .................. S5

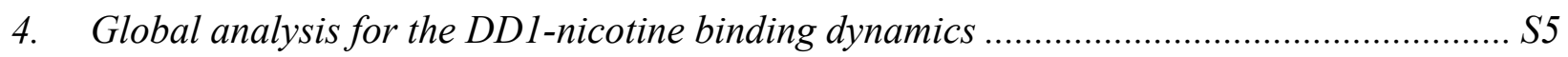

5. Binding isotherm and equilibrium constant determination for DD1-nicotine binding. ....... S6

6. DD1-nicotine binding dynamics in artificial saliva ................................................. 8

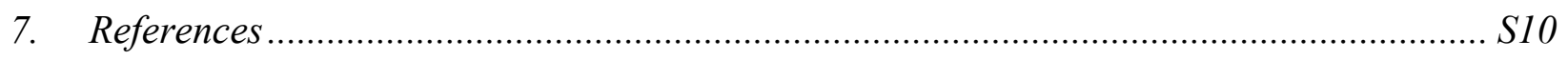




\section{Experimental section}

\section{Materials:}

DD1 was synthesized and purified following the reported protocols. ${ }^{1}$ Guest compounds (S)-(-)-nicotine, 99\% and choline chloride, $>98 \%$ were purchased from Alfa Aesar. Artificial saliva materials, methyl 4-hydroxybenzonate (Sigma-Aldrich), sodium carboxymethyl cellulose (Sigma-Aldrich), potassium chloride (Anachemia), calcium chloride (Bio Basic Canada), potassium dihydrogen phosphate (ACP Chemical), and magnesium chloride (EMD) were used as received. Dibasic sodium phosphate (Anachemia), monobasic sodium phosphate (ACP Chemicals), potassium hydroxide (EMD), sodium hydroxide (Aldrich) and hydrochloric acid (Anachemia, $4.0 \mathrm{~N}$ ) were used without further purification.

\section{Sample Preparation:}

All solutions were prepared using deionized water (Barnstead NANOpure deionizing systems, $\geq 17.8 \mathrm{M} \Omega \mathrm{cm}$ ) and the $\mathrm{pH}$ values were confirmed using a Cole Parmer Microcomputer $\mathrm{pH}-$ Vision $\mathrm{pH}$ meter. Phosphate buffer $(10 \mathrm{mM}, \mathrm{pH} 6.3)$ was prepared using dibasic sodium phosphate and monobasic sodium phosphate, the $\mathrm{pH}$ was adjusted with $1 \mathrm{M} \mathrm{NaOH} / \mathrm{HCl}$. $\mathrm{HCl}$ solutions of $\mathrm{pH} 2.2$ and $\mathrm{NaOH}$ solutions of $\mathrm{pH} 12.1$ were prepared from a $4.0 \mathrm{~N} \mathrm{HCl}$ stock solution and solid $\mathrm{NaOH}$, respectively. Artificial saliva ( $\mathrm{pH}$ 6.8) was prepared following the reported protocol, ${ }^{2}$ the $\mathrm{pH}$ was adjusted using $1 \mathrm{M} \mathrm{KOH}$ and the solution was then filtered. Solutions of DD1 and nicotine were made in the phosphate buffer $(10 \mathrm{mM}, \mathrm{pH} 6.3)$, hydrochloric acid (10 mM, $\mathrm{pH}$ 2.2), $\mathrm{NaOH}(10 \mathrm{mM}, \mathrm{pH}$ 12.1) and artificial saliva ( $\mathrm{pH}$ 6.8) solutions. A stock solution of choline chloride was made in $\mathrm{NaOH}(10 \mathrm{mM}, \mathrm{pH} 12.1)$. These stocks were further diluted with the corresponding buffer, acid, base, or saliva solution to afford the concentrations specified in the UV-Vis absorption, steady-state fluorescence and stopped-flow experiments. During stopped-flow experiments the concentrations of the solutions are halved due to equal volume mixing of the two solutions placed in each syringe before the measurement. For binding isotherm measurements, the nicotine solution contained the same concentration of DD1 as the DD1solution into which nicotine was titrated into, so that dilution of DD1 did not occur during the experiment. 
Equipment: DD1 purity was verified using a Waters UPLC-MS with a UV/Vis absorption detector, and a QDa detector. An Aquity UPLC BEH C18 $1.7 \mu \mathrm{M}(21$ x $50 \mathrm{~mm})$ column was used with a gradient of $90 \% \mathrm{H}_{2} \mathrm{O}(+0.1 \% \mathrm{FA}) / 10 \% \mathrm{CH}_{3} \mathrm{CN}\left(+0.1 \%\right.$ FA) to $30 \% \mathrm{H}_{2} \mathrm{O}(+0.1 \% \mathrm{FA}) / 70 \%$ $\mathrm{CH}_{3} \mathrm{CN}(+0.1 \% \mathrm{FA})$ over $5 \mathrm{~min}$ at $0.5 \mathrm{~mL} / \mathrm{min}$ flow.
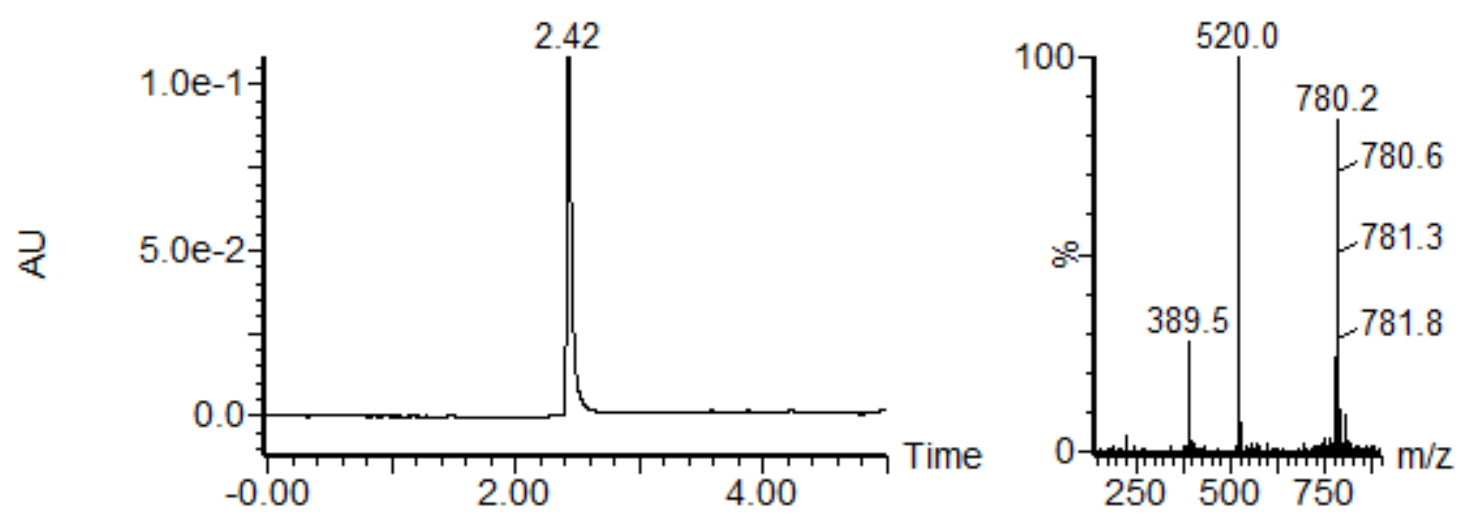

Figure S1. UPLC-MS of purified DD1 used in fluorescence and kinetic studies.

The UV-Vis absorption spectra were recorded on a Varian Cary 100 spectrophotometer. Fluorescence emission spectra were collected using a PTI QM40 fluorimeter. In the case of the DD1-nicotine system, the steady-state fluorescence emission was collected from 400-700 nm at an excitation wavelength of $375 \mathrm{~nm}$. The excitation and emission monochromator bandwidths were set to $2 \mathrm{~nm}$ for all these measurements. For the binding isotherm experiments at $\mathrm{pH} 6.3$, the emission spectra were collected between 400 and $700 \mathrm{~nm}$ at an excitation wavelength of $384 \mathrm{~nm}$.

An Applied Photophysics SX20 stopped-flow spectrometer, with a Hg-Xe vapour lamp as the excitation source, was used to measure the homodimerization and binding dynamics in phosphate buffer and artificial saliva. Two solutions were mixed within $\sim 1 \mathrm{~ms}$ in a 1:1 ratio. For the homodimerization and binding dynamics studies in the presence of phosphate buffer or artificial saliva, the samples were excited at 400 and $405 \mathrm{~nm}$ respectively, with an excitation monochromator slit width of $2 \mathrm{~mm}$ that corresponds to a bandwidth of $9.3 \mathrm{~nm}$. The emission from the sample was collected using a $515 \mathrm{~nm}$ cut-off filter. The sample temperatures were maintained at $20^{\circ} \mathrm{C}$ using a water bath. 


\section{Absorption and fluorescence measurements of DD1-nicotine at $\mathrm{pH} 2.2$ and 12.1}

The absorption spectra for DD1 at $\mathrm{pH} 2.2$ is red shifted in the presence of nicotine due to the binding of nicotine to DD1 monomer (Figure S2).

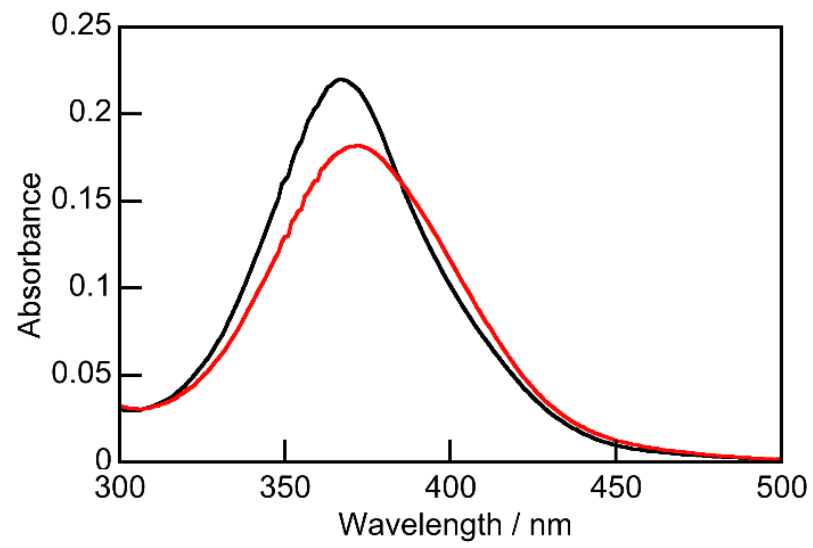

Figure S2. Absorption spectra for $8.3 \mu \mathrm{M}$ DD1 in the absence (black) and presence (red) of 167 $\mu \mathrm{M}$ nicotine at $\mathrm{pH} 2.2$.

The absorption and fluorescence spectra (Figure S3) of DD1 in the absence and presence of nicotine at $\mathrm{pH} 12.1$ show very minor changes confirming that the binding of nicotine to DD1 at this $\mathrm{pH}$ is minimal.
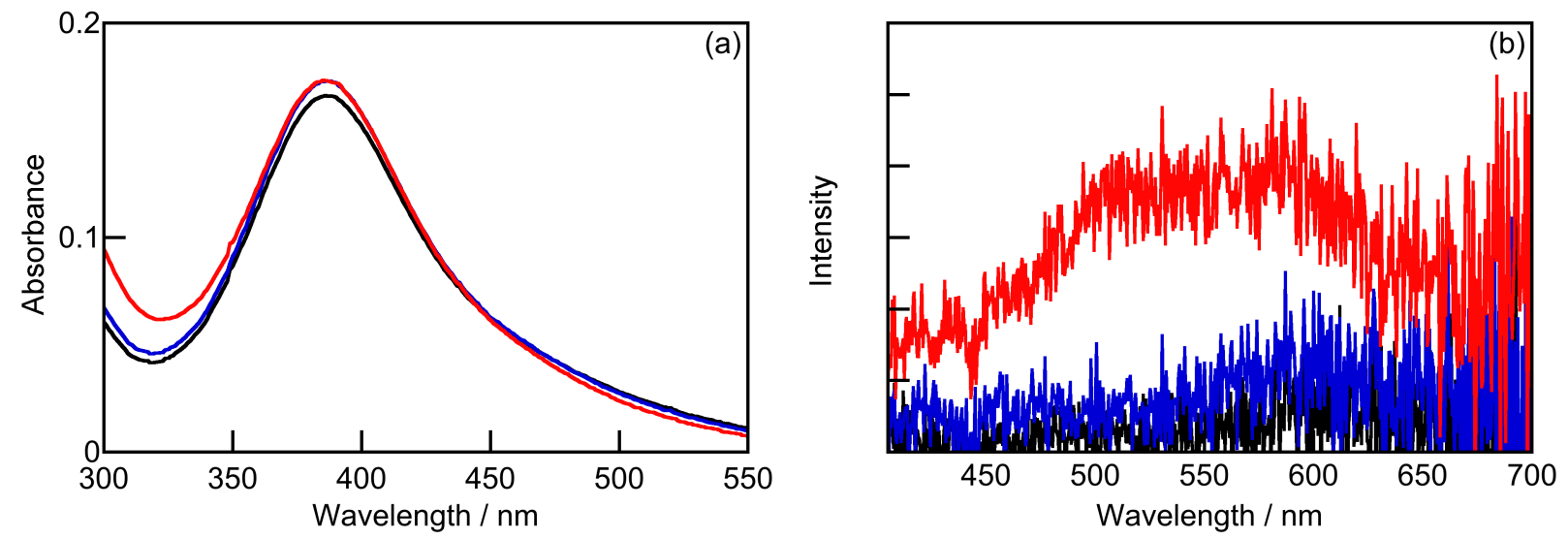

Figure S3. Absorption (a) and fluorescence (b) spectra for $10 \mu \mathrm{M}$ DD1 in the absence (black) and presence of 500 (blue) and $2000 \mu \mathrm{M}$ (red) nicotine at $\mathrm{pH} 12.1$. 


\section{Control experiment for DD1 photostability during stopped-flow experiments}

Any photoisomerization of the HSP chromophore in DD1 during the timescale of the experiment will result in a decrease in the fluorescence intensity over time due to the formation of the cis-isomer, that has a lower fluorescence quantum yield compared to the trans-isomer. ${ }^{3-4}$ Mixing two solutions of DD1 (100 $\mu \mathrm{M}$ each) resulted in constant fluorescence emission from the sample over the time period of the experiment (Figure S4), indicating that there is no photoisomerization of the HSP chromophore happening during the timescale of the experiment.

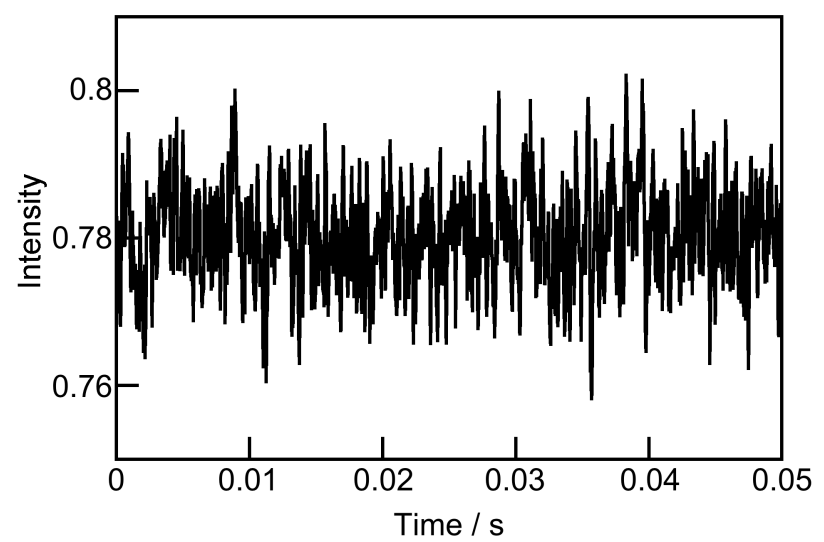

Figure S4. Stopped-flow trace for the mixing of DD1 solutions $(100 \mu \mathrm{M})$ in phosphate buffer at $\mathrm{pH} 6.3$.

\section{Global analysis for the DD1-nicotine binding dynamics}

The determination of association and dissociation rate constants for the homodimerization of DD1 enabled the use of global analysis of the stopped-flow kinetics for DD1-nicotine binding using the model shown in Scheme S1. The data from four independent experiments were analyzed simultaneously using this model. The $k_{a s}$ and $k_{d i s}$ values obtained from the analysis of homodimerization kinetics were fixed. The concentrations of DD1 and DD1 2 at the initial time $(\mathrm{t}=$ 0) required for the global analysis were calculated from the homodimerization equilibrium constant, $K_{D D 1}$ using the Micromath Scientist 3 software. The values for the $k_{a s}, k_{d i s}$ and the initial concentrations were varied between their error limits and several values for $k_{11}^{+}$and $k_{11}^{-}$were recovered. These recovered values were averaged and standards deviations were calculated to obtain the reported $k_{11}^{+}$and $k_{11}^{-}$and error values (Table 1 ). 


$$
\begin{gathered}
\mathrm{DD}_{2} \underset{\boldsymbol{k}_{a s}}{\stackrel{\boldsymbol{k}_{\text {dis }}}{\rightleftarrows}} \text { 2DD1 } \\
\mathrm{DD} 1+\text { nicotine } \frac{\boldsymbol{k}_{\boldsymbol{l 1}}^{+}}{\stackrel{\boldsymbol{k}_{\boldsymbol{l 1}}^{-}}{\rightleftarrows}} \text { DD1-nicotine }
\end{gathered}
$$

Scheme S1. Global analysis model used in the fitting of the stopped-flow kinetics for DD1nicotine binding.

The $k_{11}^{+}$and $k_{11}^{-}$values were determined for DD1-nicotine complex formation in phosphate buffer (Figure 3). A slight positive deviation was observed in the beginning of the residuals plot at higher nicotine concentrations. This deviation is due to minor processes happening in the system at high nicotine concentrations, which are not accounted for in the model used for the global analysis. The degree of interference of these unaccounted processes can be established by comparing the equilibrium constants obtained from kinetic and binding isotherm experiments. The larger the contribution of the unaccounted processes, the larger the difference between these two equilibrium constant values. Within experimental errors, the values for $K_{11}$ are the same when determined from the kinetics and binding isotherm experiments, indicating that the accuracy of $k_{11}^{+}$and $k_{11}^{-}$are not significantly affected by the presence of the minor unaccounted processes.

\section{Binding isotherm and equilibrium constant determination for DD1-nicotine binding.}

The equilibrium binding constants for nicotine binding to DD1 in phosphate buffer and artificial saliva were determined from the fit of the respective binding isotherms (Figure S5). The fluorescence emission spectra for DD1 in the presence of varying concentrations of nicotine were integrated between 500 and $700 \mathrm{~nm}$ to obtain the fluorescence intensity values. These integrated intensities were normalized with respect to the emission from DD1 in the absence of nicotine. The Scientist 3 software from Micromath was used to numerically fit the binding isotherm data. The model used in the analysis of binding isotherms considers the dimerization of DD1 and the binding of nicotine to DD1. The variables and the parameters used in the fitting routine are defined as follows: The independent variables defined by the total concentrations of nicotine and DD1 are 
denoted as $[\mathrm{N}]_{T}$ and $[\mathrm{DD} 1]_{T}$, respectively. The equilibrium concentrations of DD1, $\mathrm{DD} 1_{2}$, nicotine and DD1-nicotine 1:1 complex are denoted by $[\mathrm{DD} 1]_{e q},\left[\mathrm{DD} 1_{2}\right]_{e q},[\mathrm{~N}]_{e q}$ and $[\mathrm{DD} 1-\mathrm{N}]_{e q}$ respectively. These equilibrium concentrations and the fluorescence intensity, $I$, were defined as dependent variables in the model. The ratio of the fluorescence intensity of DD1 in the absence of nicotine $\left(I_{0}\right)$ to the total DD1 concentration represented as R, the ratio of emission efficiencies of the DD1 in the complex and in phosphate buffer or artificial saliva represented as $C_{11}$, and the equilibrium constants for homodimerization of DD1 and nicotine binding to DD1 monomer denoted by $K_{\mathrm{DD} 1}$ and $K_{11}$ respectively were defined as parameters. The following model was used in the analysis:

$$
\begin{aligned}
& {\left[\mathrm{DD} 1_{2}\right]_{e q}=K_{\mathrm{DD} 1} \times[\mathrm{DD} 1]_{e q}^{2}} \\
& {[\mathrm{DD} 1-N]_{e q}=K_{11} \times[\mathrm{DD} 1]_{e q} \times[N]_{e q}} \\
& {[\mathrm{DD} 1]_{e q}=[\mathrm{DD} 1]_{T}-2 \times\left[\mathrm{DD} 1_{2}\right]_{e q}-[\mathrm{DD} 1-\mathrm{N}]_{e q}} \\
& {[\mathrm{~N}]_{e q}=[\mathrm{N}]_{T}-[\mathrm{DD} 1-\mathrm{N}]_{e q}} \\
& I=\mathrm{R} \times\left([\mathrm{DD} 1]_{e q}+C_{11} \times[\mathrm{DD} 1-\mathrm{N}]_{e q}\right)
\end{aligned}
$$

where

$$
\mathrm{R}=\frac{I_{0}}{[\mathrm{DD} 1]_{T}}
$$

The concentration constraints defined for the dependent variables were:

$$
\begin{aligned}
& 0<[\mathrm{DD} 1]_{e q}<[\mathrm{DD} 1]_{T} \\
& 0<\left[\mathrm{DD} 1_{2}\right]_{e q}<\frac{[\mathrm{DD} 1]_{T}}{2} \\
& 0<[\mathrm{N}]_{e q}<[\mathrm{N}]_{T} \\
& 0<[\mathrm{DD} 1-\mathrm{N}]_{e q}<[\mathrm{DD} 1]_{T}
\end{aligned}
$$

The value of $K_{\mathrm{DD} 1}$ was fixed to the value obtained from the stopped-flow experiments (Table 1) and was varied between the error limits to get an average value and the corresponding standard deviation for $K_{11}$. 

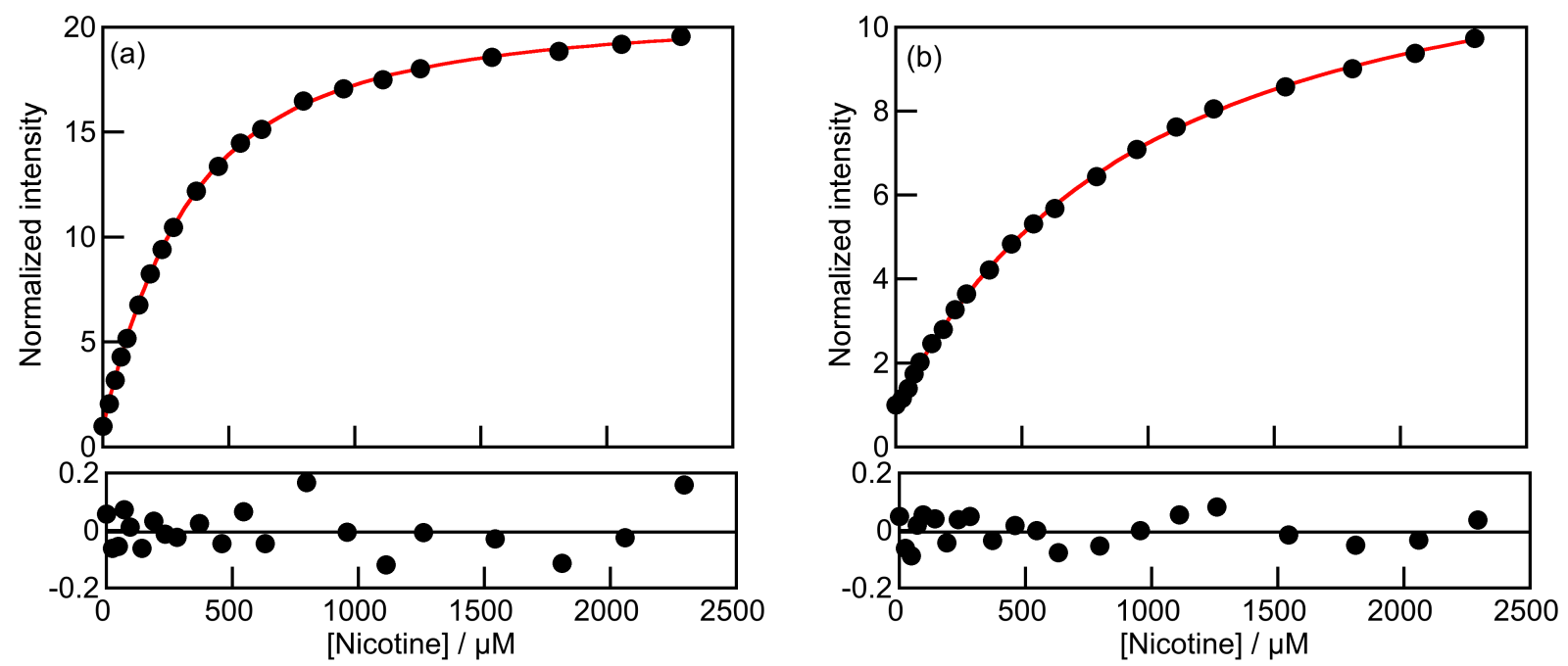

Figure S5. Fits for the dependence of the integrated fluorescence intensities on nicotine concentrations for the binding to $10 \mu \mathrm{M}$ DD1 in phosphate buffer (a) and artificial saliva (b). Residuals between the experimental data and calculated fits are shown in the bottom panels. The fluorescence intensity was normalized to the intensity in the absence of nicotine.

\section{DD1-nicotine binding dynamics in artificial saliva}

The homodimerization kinetics for $\mathrm{DD} 1_{2}$ were measured by the mixing of $\mathrm{DD} 1_{2}$ in artificial saliva with a solution of artificial saliva on the stopped-flow. Artificial saliva exhibited fluorescence in the absence of DD1 and this fluorescence was quenched by DD1. As a result, the initial fluorescence $(t=0)$ for the stopped-flow traces for artificial saliva in the presence of DD1 was lower than that of artificial saliva with no DD1. This led to an initial intensity offset for the stopped-flow kinetics measured for $\mathrm{DD}_{2}$ homodimerization. This off-set does not affect the determination of the observed rate constant. For clarity, the kinetic traces in Figure S6a are offset on the y-scale. Experimental data from 4 individual experiments were averaged and fit to equation 1 in the paper to obtain $k_{\text {as }}$ and $k_{\text {dis }}$ (Figure $\left.\mathrm{S} 6 \mathrm{~b}\right)$. 

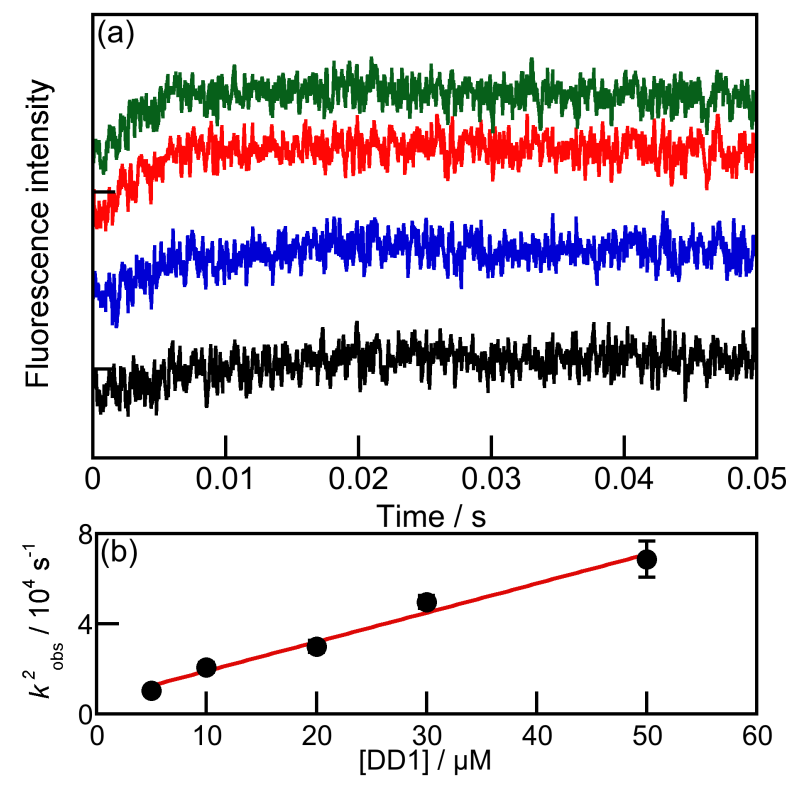

Figure S6. (a) Kinetics for the dilution of DD1 ([DD1 $]_{\text {final }}=5 \mu \mathrm{M}-$ black, $10 \mu \mathrm{M}-$ blue, $30 \mu \mathrm{M}$ - red and $50 \mu \mathrm{M}$ - green) with artificial saliva at $\mathrm{pH}$ 6.3. The traces are offset on the $\mathrm{y}$-scale for clarity. (b) Fit to equation 1 for the dependence of $k_{o b s}^{2}$ ( 4 independent experiments; errors correspond to standard deviations) on the DD1 concentration.

Qualitatively the kinetics for nicotine binding to DD1 in saliva (Figure S7) followed the same trend as for the kinetics in buffer (Figure 3b). The larger errors for $k_{11}^{+}$and $k_{11}^{-}$are a reflection of the larger errors for the rate constants recovered from the $\mathrm{DD} 1_{2}$ homodimerization kinetics. The deviations in the residuals plots at high nicotine concentrations were also observed. In this case, the $K_{11}$ value calculated from the kinetic data is lower by a factor of 2 compared to the value obtained from the binding isotherm. This discrepancy is either due to systematic deviations for the $k_{\text {as }}$ and $k_{\text {dis }}$ measured from the kinetics of $\mathrm{DD} 1_{2}$ disassembly or due to the presence of other complexes between DD1 and components of the artificial saliva. However, the trends observed in saliva for the homodimerization kinetics $\left(k_{11}^{+}\right.$and $\left.k_{11}^{-}\right)$and nicotine binding kinetics to DD1 $\left(k_{\mathrm{as}}\right.$ and $\left.k_{\mathrm{dis}}\right)$ are the same as the trends for these kinetics in buffer. 

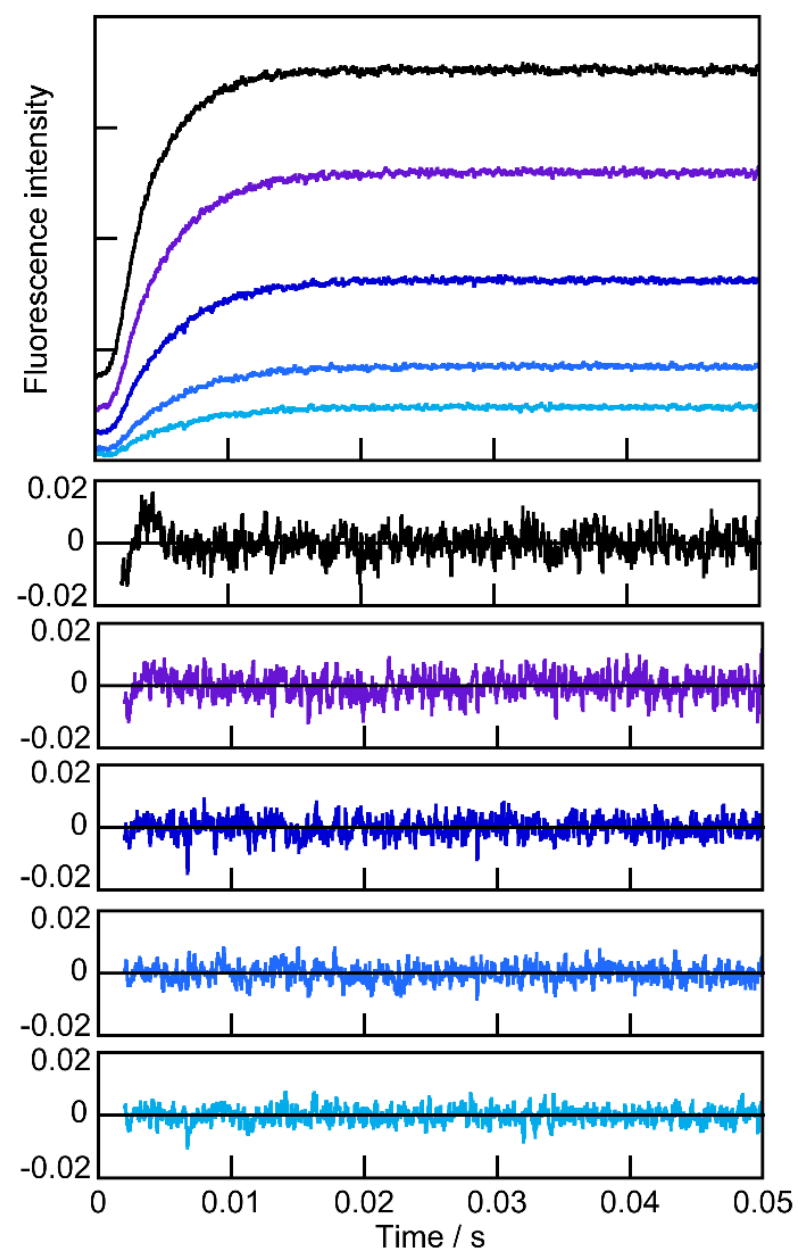

Figure S7. (a) Kinetics for the mixing of DD1 $(10 \mu \mathrm{M}, \mathrm{pH} 6.8)$ in artificial saliva with different concentrations of nicotine for one independent experiment. (b) Residuals obtained for the data in (a) from the global analysis of 21 kinetic traces from four independent experiments. For (a) and (b) the traces from the bottom to top: [nicotine $]_{\text {final }}=94,188,375,750$ and $1500 \mu \mathrm{M}$.

\section{References}

1. Beatty, M. A.; Borges-González, J.; Sinclair, N. J.; Pye, A. T.; Hof, F., Analyte-Driven Disassembly and Turn-On Fluorescent Sensing in Competitive Biological Media. J. Am. Chem. Soc. 2018, 140, 3500-3504.

2. Alshali, R. Z.; Salim, N. A.; Satterthwaite, J. D.; Silikas, N., Long-term Sorption and Solubility of Bulk-fill and Conventional Resin-composites in Water and Artificial Saliva. J. Dent. 2015, 43, 1511-1518.

3. Steiner, U.; Abdel-Kader, M. H.; Fischer, P.; Kramer, H. E. A., Photochemical cis/trans Isomerization of a Stilbazolium Betaine. A Protolytic/photochemical Reaction Cycle. J. Am. Chem. Soc. 1978, 100, 3190-3197. 
4. Tang, H.; Thomas, S. S.; Wolf, L.; Natarajan, P.; Ko, Y. H.; Wilson, J.; Kim, K.; Bohne, C., Nonlinear Dependence on $\mathrm{Na}+$ Ions for the Binding Dynamics of Cucurbit[6]uril with the trans-1-Methyl-4-(4-hydroxystyryl)pyridinium Cation. J. Phys. Chem. B 2020, 124, 10219-10225. 